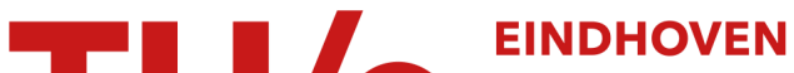 UNIVERSITY OF TECHNOLOGY
}

\section{Cardiac mechanoenergetics replicated by cross-bridge model}

\section{Citation for published version (APA):}

Vendelin, M., Bovendeerd, P. H. M., Arts, M. G. J., Engelbrecht, J., \& Campen, van, D. H. (2000). Cardiac mechanoenergetics replicated by cross-bridge model. Annals of Biomedical Engineering, 28(6), 629-640. https://doi.org/10.1114/1.1305910

DOI:

10.1114/1.1305910

Document status and date:

Published: 01/01/2000

\section{Document Version:}

Publisher's PDF, also known as Version of Record (includes final page, issue and volume numbers)

\section{Please check the document version of this publication:}

- A submitted manuscript is the version of the article upon submission and before peer-review. There can be important differences between the submitted version and the official published version of record. People interested in the research are advised to contact the author for the final version of the publication, or visit the $\mathrm{DOI}$ to the publisher's website.

- The final author version and the galley proof are versions of the publication after peer review.

- The final published version features the final layout of the paper including the volume, issue and page numbers.

Link to publication

\section{General rights}

Copyright and moral rights for the publications made accessible in the public portal are retained by the authors and/or other copyright owners and it is a condition of accessing publications that users recognise and abide by the legal requirements associated with these rights.

- Users may download and print one copy of any publication from the public portal for the purpose of private study or research.

- You may not further distribute the material or use it for any profit-making activity or commercial gain

- You may freely distribute the URL identifying the publication in the public portal.

If the publication is distributed under the terms of Article $25 f a$ of the Dutch Copyright Act, indicated by the "Taverne" license above, please follow below link for the End User Agreement:

www.tue.nl/taverne

Take down policy

If you believe that this document breaches copyright please contact us at:

openaccess@tue.nl

providing details and we will investigate your claim. 


\title{
Cardiac Mechanoenergetics Replicated by Cross-Bridge Model
}

\author{
Marko Vendelin, ${ }^{1}$ Peter H. M. Bovendeerd, ${ }^{2}$ Theo Arts, ${ }^{2,3}$ Jüri Engelbrecht, ${ }^{1}$ \\ AND DICK H. VAN CAMPEN ${ }^{2}$ \\ ${ }^{1}$ Institute of Cybernetics at Tallinn Technical University, Akadeemia 21, 12618 Tallinn, Estonia and ${ }^{2}$ Department of Mechanical \\ Engineering, Eindhoven University of Technology, Eindhoven, The Netherlands and ${ }^{3}$ Department of Biophysics, Maastricht \\ University, Maastricht, The Netherlands
}

(Received 10 June 1999; accepted 28 April 2000)

\begin{abstract}
The aim of this work is to reproduce the experimentally measured linear dependence of cardiac muscle oxygen consumption on stress-strain area using a model, composed of a three-state Huxley-type model for cross-bridge interaction and a phenomenological model of $\mathrm{Ca}^{2+}$-induced activation. By selecting particular cross-bridge cycling rate constants and modifying the cross-bridge activation model, we replicated the linear dependence between oxygen consumption and stressstrain area together with other important mechanical properties of cardiac muscle such as developed stress dependence on the sarcomere length and force-velocity relationship. The model predicts that (1) the amount of the "passenger" cross bridges, i.e., cross bridges that detach without hydrolyzing ATP molecule, is relatively small and (2) ATP consumption rate profile within a beat and the amount of the passenger cross bridges depend on the contraction protocol. (C) 2000 Biomedical Engineering Society. [S0090-6964(00)00206-X]
\end{abstract}

Keywords-Heart, Stress-strain area, Oxygen consumption, Mathematical modeling, Huxley-type model.

\section{INTRODUCTION}

In studies of regional heart muscle mechanics and energetics, oxygen consumption (or ATP consumption) is often computed on the basis of the stress-strain area (SSA), the specific area in the stress-strain (SS) diagram surrounded by the end-systolic SS line, the end-diastolic SS line, and the systolic segment of the SS trajectory for a contraction. ${ }^{6} \mathrm{SSA}$ is an analog of pressure-volume area (PVA) and, similar to PVA, the dependency of oxygen consumption in a beat on SSA is almost linear. ${ }^{14,27}$ In spite of the large amount of experimental evidence supporting the linear dependency between oxygen consumption and SSA, the computations performed using the Huxley-type models have always predicted a nonlinear relationship between these two variables. In isometric contraction, calculated ATP consumption to SSA ratio was found to decrease as a function of sarcomere

Address for correspondence: Marko Vendelin, Institute of Cybernetics, Akadeemia 21, 12618 Tallinn, Estonia. Electronic mail: markov@ioc.ee length. ${ }^{29,31}$ In isotonic contraction, the computed SSA to ATP consumption ratio was decreasing with an increase in the applied afterload. ${ }^{31}$ Thus, the current theoretical understanding of muscle contraction cannot replicate the fundamental property of cardiac muscle, that oxygen consumption and SSA are linearly related. The importance of resolving this contradiction was outlined by several reviews. In 1985, ${ }^{11}$ Gibbs and Chapman conclude: "In summary, however, we appear to be faced by a paradox. Although many features of the cardiac results imply that there is a stoichiometric relation between ATP consumption and the manifestation of pressure-volume (or force-length) potential energy, the stoichiometric slippage essentially built into all models of muscle contraction ... predicts that this should not be the case." Ten years later, in 1995, Gibbs and Barclay ${ }^{10}$ stated the following: "It does seem essential that cardiac cross-bridge models can replicate both the time varying elastance data and also account for the linear correlation between pressure-volume area and oxygen consumption."

One way to resolve the contradiction between the mentioned theoretical studies and experimental measurements is to assume the existence of so-called "passenger' cross-bridges, i.e., cross bridges that are forced to detach from actin without consuming ATP molecule. This assumption has been applied successfully for the analysis of ATP consumption at high shortening velocities of the muscle fibers ${ }^{4}$. According to simulations of Cooke et al., ${ }^{4}$ about $80 \%$ of all cross bridges do not consume ATP during a cycle at high sarcomere shortening velocities. This means that the ATP molecule is hydrolyzed only once per five actomyosin cycles. However, contrary to isotonic contraction, the presence of " "passenger' cross bridges has a small probability in isometric contraction, where the sarcomere shortening velocity is too low to drag the attached cross bridges into the region of cross-bridge detachment without consuming an ATP molecule. 
TABLE 1. Model parameters.

\begin{tabular}{|c|c|c|c|c|}
\hline Element & Parameter & Dimension & Value & Source \\
\hline \multirow[t]{5}{*}{ Passive stress } & $a_{0}$ & $\mathrm{kPa}$ & 0.5 & Bovendeerd et al. ${ }^{1}$ \\
\hline & $a_{1}$ & & 3 & Bovendeerd et al. ${ }^{1}$ \\
\hline & $a_{2}$ & & 6 & Bovendeerd et al. ${ }^{1}$ \\
\hline & $a_{3}$ & & 3 & Bovendeerd et al. ${ }^{1}$ \\
\hline & $a_{4}$ & $\mathrm{kPa}$ & 150 & \\
\hline \multirow[t]{2}{*}{ Series elasticity } & $I_{\mathrm{se}}^{\mathrm{N}}$ & $\mu \mathrm{m}$ & 0.02 & \\
\hline & $K_{s e}$ & $\mu \mathrm{m}^{-1}$ & 75 & \\
\hline \multirow[t]{15}{*}{ Contractile element } & $K$ & $R T \mathrm{~nm}^{-2}$ & 0.56 & Pate and Cooke ${ }^{24}$ \\
\hline & $G_{W}$ & $R T$ & 0 & \\
\hline & $G_{S 1}$ & $R T$ & $K z^{2}-18.4$ & \\
\hline & $G_{S 2}$ & $R T$ & $K z^{2}-20.7$ & \\
\hline & $d$ & $\mathrm{~nm}$ & 36 & Eisenberg et al. $^{9}$ \\
\hline & $m$ & $\mathrm{~mol} \mathrm{~m}^{-3}$ & 0.18 & van der Velden et al. ${ }^{33}$ \\
\hline & $c_{1}$ & $\mathrm{~s}^{-1}$ & 27.62 & \\
\hline & $c_{2 M X}$ & $s^{-1}$ & 4.49 & \\
\hline & $c_{2 F}$ & $\mathrm{~s}^{-1}$ & 11.22 & \\
\hline & $Q$ & & 0.065 & \\
\hline & $T_{P}$ & s & 0.025 & Callewaert $^{3}$ \\
\hline & $T_{D 0}$ & s & 0.1 & \\
\hline & $T_{D 1}$ & & 1.5 & \\
\hline & $I_{\min }$ & $\mu \mathrm{m}$ & 1.6 & Jewell $^{18}$ \\
\hline & $I_{\max }$ & $\mu \mathrm{m}$ & 2.3 & \\
\hline
\end{tabular}

The goal of this study is to replicate the measured linear relation between ATP consumption and SSA in the isometric and shortening contractions using a model, composed of the Huxley-type model for cross-bridge interaction and a phenomenological model of $\mathrm{Ca}^{2+}$-induced activation (the Huxley-type model for short). The secondary goal is to predict the relative amount of the "passenger" cross bridges required to obtain the relation between ATP consumption and SSA. Additional requirements for the model were set by the intended use of the model in the future studies of the regional discrepancies between the mechanical contraction and oxygen supply in the heart wall. Since these studies will require detailed description of the relation between energetics and mechanics only, we modeled the excitation-contraction coupling and basal metabolism by simple phenomenological relations. Moreover, in view of these studies we already use a three-dimensional description of the constitutive properties of cardiac tissue.

In the present study, we found the model parameters by minimizing the difference between the selected measurements and model solution. During the optimization process, we took into account thermodynamic relations between the parameter values to compose the model which is self-consistent with the thermodynamical laws. ${ }^{9,13}$ Then, without any additional optimization, we compared model predictions with experimental data not used in the parameter estimation, in order to assess the predictive quality of our model.

\section{MODEL DESCRIPTION}

In the present study we modeled the cardiac tissue as a set of contractile fibers, mutually connected by a passive tissue network. The contractile fibers were modeled as a contractile element in series with an elastic element. The contractile element was described by the selfconsistent sliding filament model. ${ }^{9,13}$ The series elastic element, reflecting the compliance within thick and thin filaments in the nonoverlap zone, was characterized by an exponential relationship between stress and strain. The compliance within the overlap zone of the filaments ${ }^{5}$ has been neglected in this work. Finally, the threedimensional passive tissue network was assumed to behave as a nonlinearly elastic, anisotropic material. The values of the constants used in the model are shown in Table 1.

\section{Constitutive Behavior}

The total Cauchy stress $\boldsymbol{\sigma}$ developed in myocardial tissue is divided into (a) the uniaxial active stress $\sigma_{a}$ generated by the contractile element parallel to the muscle fiber direction $\boldsymbol{e}_{\boldsymbol{f}}$ and (b) the three-dimensional passive stress $\boldsymbol{\sigma}_{\boldsymbol{p}}$ resulting from the tissue deformation (parallel elastic element)

$$
\boldsymbol{\sigma}=\sigma_{a} e_{f} e_{f}+\boldsymbol{\sigma}_{p}
$$




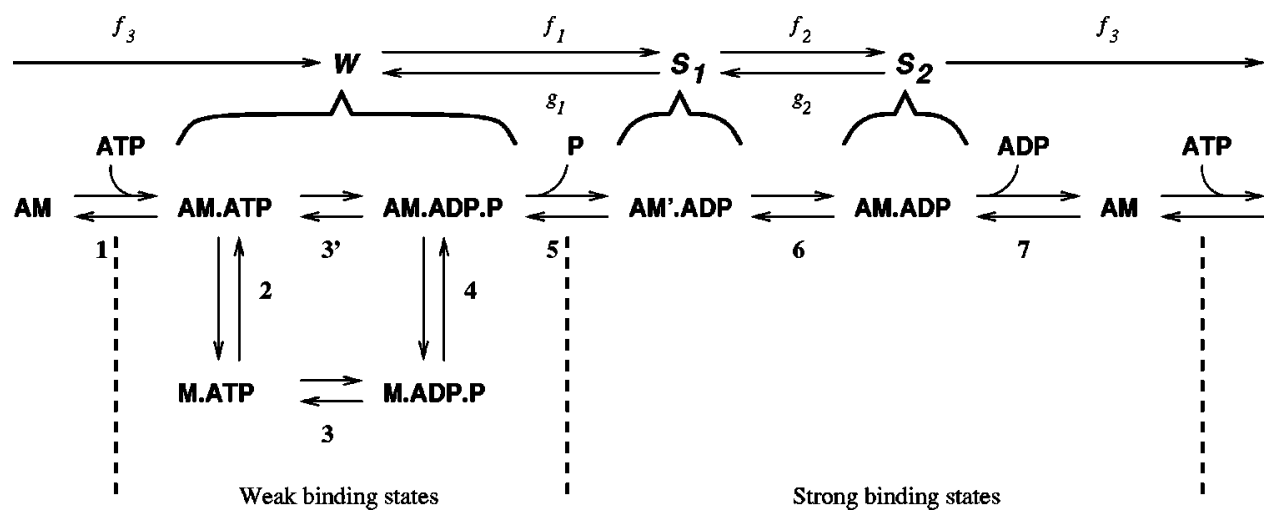

FIGURE 1. Kinetic scheme of actin (A) and myosin (M) interaction (lower part) and its simplification as used in the model (upper part). States which include only $M$ in the scheme present the states where myosin head is not attached to actin filament.

\section{Three-Dimensional Passive Element}

The passive stress $\boldsymbol{\sigma}_{\boldsymbol{p}}$ is determined by a strain energy function $W(\mathbf{E})$ that relates the second Piola-Kirchhoff stress tensor $\mathbf{S}$ to the Green-Lagrange strain tensor $\mathbf{E}$ :

$$
\mathbf{S}=\partial W(\mathbf{E}) / \partial \mathbf{E}
$$

The strain energy is taken from Rijcken et al. ${ }^{25}$

$$
\begin{aligned}
W(\mathbf{E})= & a_{0}\left\{\exp \left[a_{1} I_{E}^{2}+a_{2} I I_{E}+a_{3}\left(\boldsymbol{e}_{f} \cdot \mathbf{E} \cdot \boldsymbol{e}_{f}\right)^{2}\right]-1\right\} \\
& +a_{4}[\operatorname{det}(2 \mathbf{E}+\mathbf{I})-1]^{2},
\end{aligned}
$$

where $a_{i}(i=0,1,2,3,4)$ are material parameters,

$$
\begin{gathered}
I_{E}=\operatorname{trace}(\mathbf{E}), \\
I I_{E}=\frac{1}{2}\left[\operatorname{trace}\left(\mathbf{E} \cdot \mathbf{E}^{c}\right)-I_{E}^{2}\right],
\end{gathered}
$$

and $\mathbf{I}$ is the unity tensor. The passive Cauchy stress $\boldsymbol{\sigma}_{\boldsymbol{p}}$ is found from $\mathbf{S}$ according to

$$
\boldsymbol{\sigma}_{p}=\frac{1}{\operatorname{det}(\mathbf{F})} \mathbf{F} \cdot \mathbf{S}^{c} \cdot \mathbf{F}^{c},
$$

where $\mathbf{F}$ is the deformation gradient tensor and index $c$ denotes a tensor conjugate.

\section{Series Elastic Element}

The series elastic element is described by the commonly used exponential law. ${ }^{23,31}$ The Cauchy stress developed by the series elastic element $\sigma_{\text {se }}$ is

$$
\sigma_{\mathrm{se}}=P_{\mathrm{se}}\left[\exp \left(K_{\mathrm{se}} l_{\mathrm{se}}\right)-1\right]
$$

where $l_{\mathrm{se}}$ is the length of the series elastic element,

$$
P_{\mathrm{se}}=P_{N} /\left[\exp \left(K_{\mathrm{se}} l_{\mathrm{se}}^{N}\right)-1\right]
$$

and $P_{N}$ is normalization stress equal to $100 \mathrm{kPa}, l_{\mathrm{se}}^{N}$ is the series elastic element length at a stress of $P_{N}$, and $K_{\mathrm{se}}$ is the element constant. With the used parameter values, $\sigma_{\mathrm{se}}$ is almost a linear function of $l_{\text {se }}$ in the simulated conditions. Due to the composition of the series elastic and contractile elements in the contractile fibers, the length of the contractile element $l_{c}$ is smaller than the sarcomere length $l_{s}$ by $l_{\mathrm{se}}:{ }^{31}$

$$
l_{s}=l_{c}+l_{\mathrm{se}} .
$$

\section{Contractile Element}

It is widely accepted that mechanical force is produced as a result of cyclic interaction of myosin heads with actin filaments. ${ }^{15,16,26}$ During this interaction, the actomyosin complex is transfered from weak binding states to strong binding states and back to the weak states $^{26}$ (Fig. 1). Only the strong binding states produce mechanical force. The transformation of the actomyosin complex is driven by the free energy of ATP hydrolysis to ADP and inorganic phosphate $\mathrm{Pi} .{ }^{12,26,28}$

Several reactions presented in Fig. 1 are much faster than the rest. Namely, reactions (2) and (4) are in rapid equilibrium. $^{12}$ Thus, states AM.ATP and M.ATP form only one "functional" state, just as states AM.ADP.P and M.ADP.P do. In vivo, ATP attachment to AM is also very fast ${ }^{12,28}$ effectively reducing the amount of the cross bridges in this state to a negligible value. Thus, to describe actomyosin interaction one has to focus only on four states: AM.ATP + M.ATP, AM.ADP.P + M.ADP.P, $\mathrm{AM}^{\prime}$.ADP, and AM.ADP. For the sake of simplicity, we will assume that states AM.ATP, M.ATP, AM.ADP.P, and M.ADP.P may be presented by only 
one state. This will reduce the model to a three-state model with two strong binding states $\left(S_{1}\right.$ and $\left.S_{2}\right)$ and one weak binding state $W$ with the rate constants $f_{i}$ and $g_{i}$ (see upper part of Fig. 1). The only reaction which is considered to be irreversible, is the cross-bridge detachment $S_{2} \rightarrow W$ due to the high rate of the forward reaction 1 compared to the rate of reverse reaction 7 in normal conditions.

The force produced by the attached cross bridge in states $S_{1}$ and $S_{2}$ is assumed to be elastic and depends linearly on the axial distance $z$ along the myosin and actin filaments between the equilibrium position of the myosin head and the nearest actin binding site. The equilibrium position and the elasticity constant for states $S_{1}$ and $S_{2}$ are taken to be equal. Since the force $F_{\alpha}$ developed by the cross bridge in state $\alpha$ and the free energy $G_{\alpha}$ of the cross bridge obey $F_{\alpha}=\partial G_{\alpha} / \partial z$, the linear dependence of $F$ on $z$ leads to a parabolic dependence of the free energy on $z .^{13}$

Taking into account the distribution of the cross bridges among the states as a function of $z$, the Cauchy stress $\sigma_{a}$ developed by the cross bridges in a halfsarcomere is, according to Zahalak and $\mathrm{Ma},{ }^{35}$ as follows:

$$
\begin{aligned}
\sigma_{a}= & \frac{m l_{s}}{2 d}\left(\int_{-d / 2}^{d / 2} n_{S 1} F_{S 1} d z+\int_{-d / 2}^{d / 2} n_{S 2} F_{S 2} d z\right. \\
& \left.+\int_{-d / 2}^{d / 2} n_{W} F_{W} d z\right)
\end{aligned}
$$

where $n_{W}, n_{S 1}, n_{S 2}$ are the fractions of the cross bridges in states $W, S_{1}$, and $S_{2}$, respectively; $F_{S 1}, F_{S 2}$, and $F_{W}$ is the force produced by the cross bridge in the corresponding states; $m$ is the number of cross bridges in the unit volume; and $d$ is the distance between successive actin sites to which the cross bridge can attach. According to our assumptions, the forces $F_{S 1}, F_{S 2}$ are linearly dependent on $z$ with the Hooke constant $K$, and $F_{W}$ is zero:

$$
\sigma_{a}=\frac{m l_{s} K}{2 d}\left(\int_{-d / 2}^{d / 2} n_{S 1} z d z+\int_{-d / 2}^{d / 2} n_{S 2} z d z\right)
$$

The cross-bridge attachment and detachment in the muscle fiber is governed by the following system of equations:

$$
\begin{gathered}
\frac{\partial n_{S 1}}{\partial t}=f_{1} n_{W}+g_{2} n_{S 2}-\left(g_{1}+f_{2}\right) n_{S 1}-w \frac{\partial n_{S 1}}{\partial z}, \\
\frac{\partial n_{S 2}}{\partial t}=f_{2} n_{S 1}-\left(g_{2}+f_{3}\right) n_{S 2}-w \frac{\partial n_{S 2}}{\partial z},
\end{gathered}
$$

$$
n_{W}=A-n_{S 1}-n_{S 2},
$$

where $A$ is the relative amount of activated cross bridges and $w$ is the velocity of the contractile element lengthening

$$
w=\frac{d l_{c}}{d t}=\frac{d}{d t}\left(l_{s}-l_{\mathrm{se}}\right) .
$$

According to Eq. (14), only the activated cross bridges contribute to the force development. The rate constants are functions of $z$ and the ratios of the forward and reverse reaction rates are determined by the change in the cross-bridge free energy as follows:

$$
\begin{aligned}
& f_{1} / g_{1}=\exp \left[-\left(G_{S 1}-G_{W}\right) / R T\right], \\
& f_{2} / g_{2}=\exp \left[-\left(G_{S 2}-G_{S 1}\right) / R T\right] .
\end{aligned}
$$

The activation function $A$ introduces two important cardiac muscle properties into the model: (a) the time dependence of the stress generation in a twitch and (b) the developed stress dependence on the sarcomere length. ${ }^{18}$ The muscle activation is governed by $\mathrm{Ca}^{2+}$ concentration through an intermediate state $B$ in reaction between troponin $\mathrm{C}$ and $\mathrm{Ca}^{2+}$ as follows:

$$
\frac{d A}{d t}=c_{1} B(1-A)-c_{2}\left(l_{s}\right) \frac{A}{Q+A},
$$

where $c_{2}$ is a function of sarcomere length $l_{s}$

$$
c_{2}=c_{2 M X}+c_{2 F} \frac{l_{\max }-l_{s}}{l_{s}-l_{\min }},
$$

and normalized concentration $B$ is a function of time

$$
B=\left\{\begin{array}{l}
\left(t / T_{P}\right)^{4}, \quad \text { if } t \leqslant T_{P} \\
\exp \left\{-\left[\left(t-T_{P}\right) / T_{D}\right]^{2}\right\}, \quad \text { if } t>T_{P} .
\end{array}\right.
$$

In these equations, $c_{1}, c_{2 M X}, c_{2 F}$ are the rate constants, $Q$ introduces the cooperativity of the $\mathrm{Ca}^{2+}$ binding to the troponin $\mathrm{C}^{32}$ and $l_{\min }, l_{\max }$ are the sarcomere minimum and maximum length, respectively. The characteristic duration time $T_{D}$ is dependent on $l_{s}$ and given by

$$
T_{D}=T_{D 0}\left(1+T_{D 1} \frac{l_{s}-l_{\min }}{l_{\max }-l_{\min }}\right),
$$

and the time to peak $T_{P}$ is constant. 

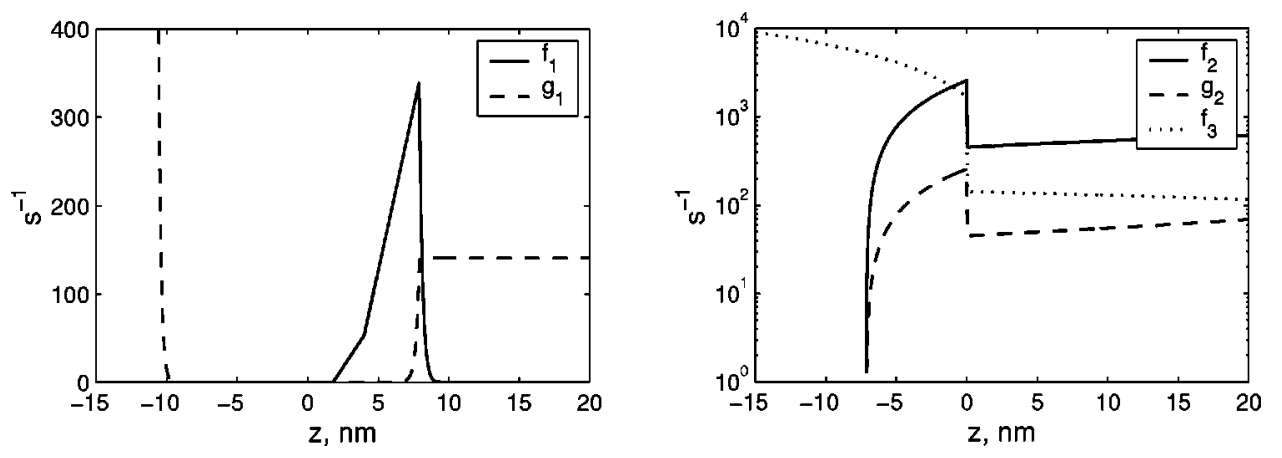

FIGURE 2. Cross-bridge cycling rates used in the model. The rates were found by optimization. Note the difference in scales of $y$ axis between the left and the right panels.

The ATP consumption rate is taken equal to the crossbridge cycling rate

$$
V_{\mathrm{ATP}}=\frac{1}{d} \int_{-d / 2}^{d / 2} f_{3}(z) n_{S 2}(z, t) d z
$$

leading to the total ATP consumption during a cycle

$$
V_{\mathrm{ATP}}^{\text {beat }}=\frac{1}{d} \int_{0}^{T} \int_{-d / 2}^{d / 2} f_{3}(z) n_{S 2}(z, t) d z d t
$$

where $T$ is the period of a beat. According to the experimental studies reviewed by Suga, ${ }^{27}$ it is reasonable to assume that ATP consumption by excitation-contraction coupling and basal metabolism is almost constant regardless of PVA in given contractile state. This assumption allowed us to compute the contractile efficiency from the cross-bridge ATP consumption only and ignore the contributions to ATP consumption by other intracellular processes.

\section{Optimization Strategy}

In this study we looked for the set of model parameters that led to the cross-bridge model which satisfied the experimentally measured linear dependency of oxygen consumption on SSA. We divided the parameters into two sets: (a) the parameters describing $\mathrm{Ca}^{2+}$-induced activation of the actomyosin complex, and (b) the rate constants of the actomyosin complex state transformation reactions. The parameters in the sets were optimized by minimization of the different residual functions.

In each optimization step for the set (b), the crossbridge cycling rate constants were specified and the set of the actomyosin activation parameters $c_{1}, c_{2 M X}, c_{2 F}$, and $Q$ were found by fitting the computed and measured active stress development in isosarcometric contraction. ${ }^{17}$
The used residual function $R_{\text {act }}$ was the difference between computed and measured stress at different sarcomere length values

$$
R_{\mathrm{act}}=\frac{1}{N} \sum_{i=1}^{N}\left[\sigma\left(l_{i}, t_{i}\right)-\sigma_{0}\left(l_{i}, t_{i}\right)\right]^{2},
$$

where $\sigma$ and $\sigma_{0}$ are computed and measured stress, respectively; $l_{i}$ is sarcomere length, and $t_{i}$ is time moment for measurement point $i$, and $N$ is the number of measurement points. The measurement points used in the optimization are shown by crosses in Fig. 3.

To find cross-bridge cycling rates $f_{1}, g_{1}, f_{2}, g_{2}$, and $f_{3}$ we predescribed the shape of these functions. Taking into account Eqs. (16) and (17), there are only three independent cross-bridge cycling rates for every $z$. The shapes of the functions are shown in Fig. 2 and were obtained as follows. Rates $f_{1}$ and $g_{1}$ were zero at negative $z$ except such values of $z$ at which $f_{1}$ was more than 1000 times smaller than $g_{1}$. At these values of $z, f_{1}$ was $1 \mathrm{~s}^{-1}$. At positive $z, f_{1}$ was piecewise linear until $g_{1}$ reached the predescribed maximum value $g_{1}(+\infty)$. Functions $g_{2}$ and $f_{3}$ were given by two linear relations each, one for positive and one for negative $z$ values. The minimal value for the rates was zero.

The cross-bridge cycling rates were varied to obtain (a) the linear dependency between $V_{\text {ATP }}^{\text {beat }}$ and SSA for isometric and shortening contractions, (b) prescribed SSA to $V_{\text {ATP }}^{\text {beat }}$ efficiency, (c) prescribed shortening velocity at $2.5 \mathrm{kPa}$ afterload in the isotonic contraction, and (d) end-systolic points in stress-strain diagram for isotonic contraction close to the maximal total stress in isometric contraction. The residual function $R_{\text {cyc }}$ which was minimized by varying the cross-bridge rates, had seven terms: 


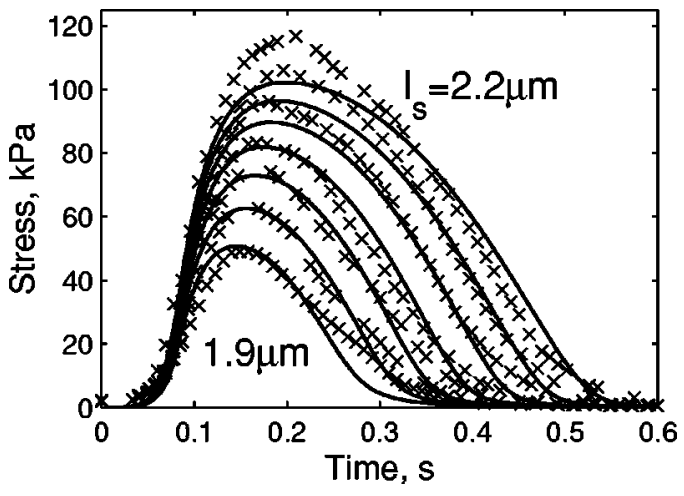

FIGURE 3. Computed (solid lines) and measured (crosses) stress during the isometric contraction as a function of time at sarcomere length of 1.9, 1.95, 2.0, 2.05, 2.1, 2.15, and 2.2 $\mu \mathrm{m}$. The computed force is compared with measurements at an extracellular $\mathrm{Ca}^{2+}$ concentration of $2 \mathrm{mM}$ (Ref. 17). The stress was computed on the basis of an undeformed fiber with a sarcomere length of $2.0 \mu \mathrm{m}$. The measurements were performed at $23-24^{\circ} \mathrm{C}$ (Ref. 17).

$$
\begin{aligned}
R_{\mathrm{cyc}}= & R_{\text {act }} / N R_{\text {act }}+\left(V_{0}-V_{0}^{\text {exp }}\right)^{2} / N R_{V 0} \\
& +\left(V_{\mathrm{ATP}}^{\text {beat }}-V_{\mathrm{ATP}}^{\mathrm{SSA}}\right)^{2} / N R_{\mathrm{ATP}}^{\text {beat }} \\
& +R_{l s} / N R_{l s}+R_{\mathrm{ATP}}^{t} / N R_{\mathrm{ATP}}^{t} \\
& +R_{\mathrm{ATP}}^{p} / N R_{\mathrm{ATP}}^{p}+R_{\mathrm{ATP}}^{\operatorname{lin}} / N R_{\mathrm{ATP}}^{\operatorname{lin}},
\end{aligned}
$$

where $R_{\text {act }}$ is defined by Eq. (24); $V_{0}$ and $V_{0}^{\exp }$ are computed and measured shortening velocity at $2.5 \mathrm{kPa}$ afterload in the isotonic contraction, respectively; $V_{\mathrm{ATP}}^{\text {beat }}$ is total ATP consumption during a cycle computed on the basis of Eq. (23) in isometric contraction at sarcomere length of $2.2 \mu \mathrm{m} ; V_{\mathrm{ATP}}^{\mathrm{SSA}}$ is total ATP consumption during a cycle computed from SSA assuming $60 \%$ contractile efficiency in isometric contraction at sarcomere length of $2.2 \mu \mathrm{m} ; R_{l s}$ is the sum of squares of differences of end-systolic points for isometric and isotonic contractions with the same end-systolic stress; $R_{\mathrm{ATP}}^{t}$ is the sum of squares of differences of computed $V_{\mathrm{ATP}}^{\text {beat }}$ for isometric and isotonic contractions with the same SSA; $R_{\mathrm{ATP}}^{p}$ is the same as $R_{\mathrm{ATP}}^{t}$ but with the differences taken between $V_{\mathrm{ATP}}^{\text {beat }}$ for isometric and physiologic contractions; $R_{\mathrm{ATP}}^{\operatorname{lin}}$ is a square of the largest deviation from linear relationship between $V_{\mathrm{ATP}}^{\text {beat }}$ and SSA computed for isometric contractions. The normalization factors $N R_{\text {act }}, N R_{V 0}, N R_{\text {ATP }}^{\text {beat }}$, $N R_{l s}, N R_{\mathrm{ATP}}^{t}, N R_{\mathrm{ATP}}^{p}$, and $N R_{\mathrm{ATP}}^{\mathrm{lin}}$ were $(50 \mathrm{kPa}){ }^{2}{ }^{2}(12$ $\mu \mathrm{m} / \mathrm{s}),{ }^{2} 1,(0.3 \mu \mathrm{m}),{ }^{2}\left(V_{\mathrm{ATP}}^{\mathrm{SSA}}\right),{ }^{2}\left(V_{\mathrm{ATP}}^{\mathrm{SSA}}\right),{ }^{2}$ and $(0.25),{ }^{2}$ respectively.

The minimization of $R_{\text {act }}$ was stopped when either $R_{\text {act }}$ or the activation parameters change was less than $0.001 \%$ after an optimization step. The optimization of $R_{\text {cyc }}$ was stopped when seven consecutive steps reduced $R_{\text {cyc }}$ by less than $1 \%$ per step.
The rate constants and the activation parameters obtained by the optimization are shown in Fig. 2 and in Table 1.

\section{Numerical Methods}

The partial differential equations were solved by the finite-difference method. The resulting system of ordinary differential equations was solved by the backwards differentiation formula that is able to treat stiff equations. The accuracy of the solution was tested by comparing different spatial discretizations and varying the tolerance of the ordinary differential equations solver. The optimization was performed using the Levenberg-Marquardt algorithm. $^{22}$

\section{RESULTS \\ Isometric Contraction}

Contraction was driven through the prescribed $B$ transient (20) followed by activation described by (18). The time course of the active force development at different preloads is presented in Fig. 3. Both the maximal developed force and the twitch duration increase with the increase of the preload. The same is true for ATP consumption rate during a cycle leading to the larger than linear increase of the total ATP consumption dependency on the sarcomere length.

\section{Shortening Contractions}

We have simulated two types of the shortening contractions: isotonic and "physiologic" contractions mimicking the sarcomere contraction during a heartbeat. Here, under the physiologic contraction we mean the isotonic contraction until the minimal sarcomere length is reached and the isometric contraction after that moment. The computed total force for the isotonic and physiologic contractions at different afterloads is shown in Fig. 4.

Since SSA is the same for isotonic and the physiologic contractions, ATP consumption during the cycle has to be the same for these contractions to achieve the same linear dependence between $V_{\text {ATP }}^{\text {beat }}$ and SSA. Indeed, the simulations show that the difference between $V_{\text {ATP }}$ computed for isotonic and the physiologic contractions is relatively small (Fig. 5).

According to several experimental studies at tissue and muscle fiber levels, the end-systolic lines for isotonic and isometric contractions in the force-length plane are relatively close to each other. The computed end-systolic lines for the isometric and isotonic contractions are compared in Fig. 6.

Good correlation was found between the computed sarcomere shortening velocity at the beginning of the shortening in isotonic contraction with the measurements 


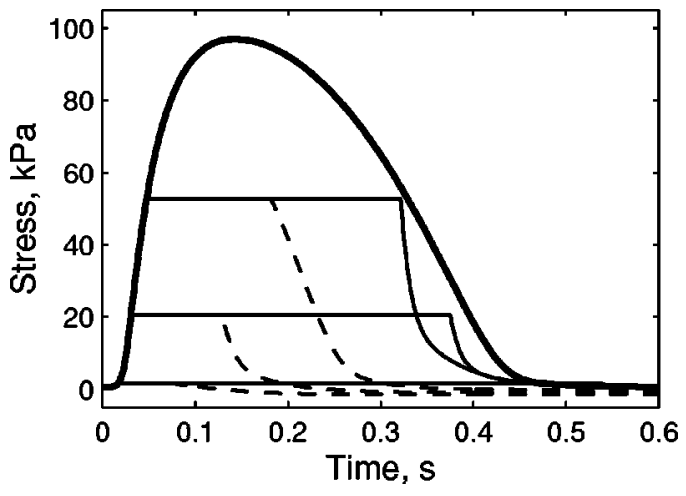

FIGURE 4. Developed stress for the isometric (bold line), isotonic (thin solid lines) and the physiologic (thin dashed lines) contractions. The fiber was prestretched by an externally applied force from the sarcomere length of 2.0 to about $2.2 \mu \mathrm{m}$. The stress was computed on the basis of an undeformed fiber with a sarcomere length of $2.0 \mu \mathrm{m}$.

by van Heuningen et al. $^{34}$ (Fig. 7), at moderate and high afterloads. At very low workloads the computed shortening velocity is larger than measured, reaching 10.7 $\mu \mathrm{m} \mathrm{s}^{-1}$ in computations opposed to measured $8 \mu \mathrm{m} \mathrm{s}^{-1}$ at zero afterload. The correlation coefficient $(r)$ was equal to 0.981 .

\section{ATP Consumption}

Our simulations replicate the linear dependency between ATP consumption and SSA for different contraction modes (Fig. 8). In isotonic and the physiologic contractions presented here, the muscle was prestretched to sarcomere length $2.17 \mu \mathrm{m}$ as in the computations presented in Fig. 6. The line fitted to ATP dependency on SSA for isometric contraction is

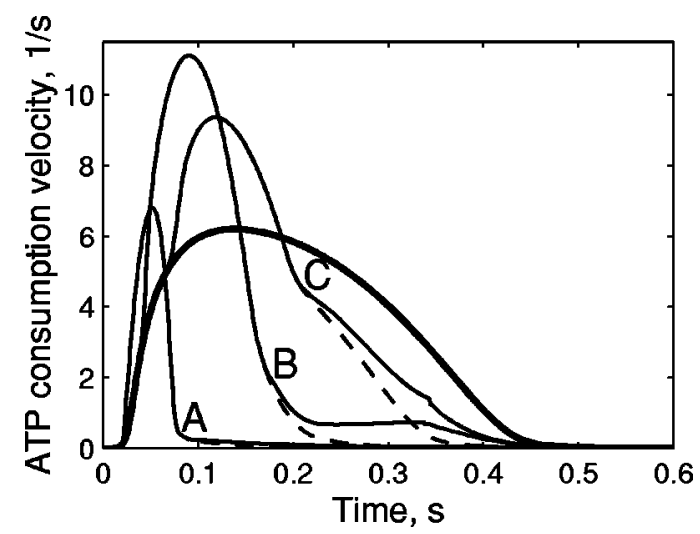

FIGURE 5. ATP consumption rate for isometric (bold line), isotonic (thin solid lines) and physiologic (thin dashed lines) contractions at afterloads of 3 (line A), 39 (line B), and 75 $\mathrm{kPa}$ (line $\mathrm{C}$ ). Note the small differences between isotonic and the physiologic contractions.

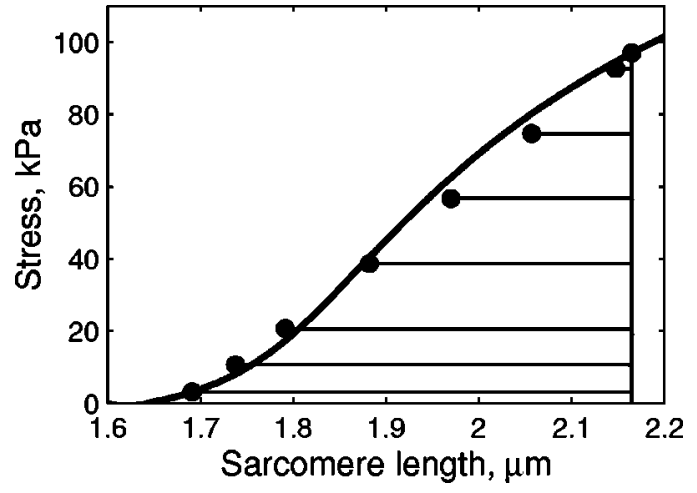

FIGURE 6. End-systolic line for the isometric contraction (bold line) and the isotonic contraction traces (thin lines) at different afterloads. Note that the end-systolic points for the isotonic contractions (points) lie close to the end-systolic line for the isometric contractions.

$$
V_{\mathrm{ATP}}^{\text {beat }}=0.046+0.142 \mathrm{~kJ}^{-1} \mathrm{~m}^{3} \mathrm{SSA},
$$

with correlation coefficient $(r)$ equal to 0.9987 and the largest deviation from this line about 0.05 . Taking into account the myosin ATPase concentration ${ }^{33}$ of $0.18 \mathrm{mM}$ or $0.18 \mathrm{~mol} \mathrm{~m}^{-3}$ and free energy change during ATP hydrolysis of $60 \mathrm{~kJ} \mathrm{~mol}^{-1},{ }^{10,27}$ the contraction efficiency calculated from the slope of the line is $65.4 \%$ and for maximal computed SSA value presented in Fig. 8 is $65.4 \%$ as well.

\section{Quick-Release Experiment}

A common approach used to study ATP consumption rate during the cycle is to perform quick-release experiments and measure the amount of consumed ATP (or $\mathrm{O}_{2}$ ) as a function of the release time. In the numerical experiment, the muscle was allowed to contract isometri-

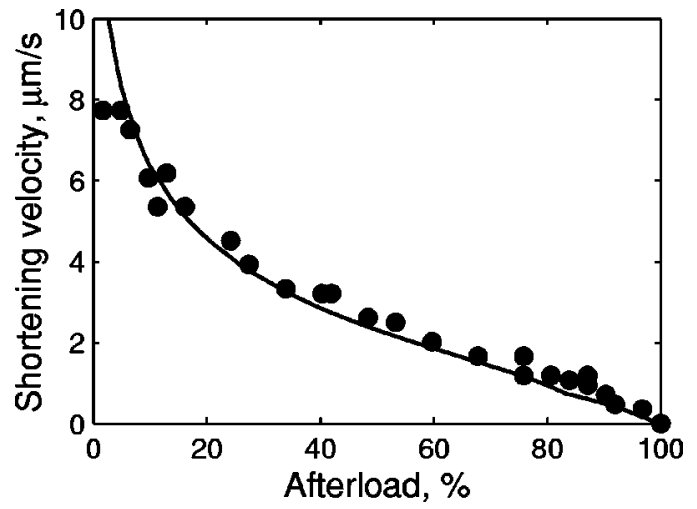

FIGURE 7. Computed (solid line) and measured by van Heuningen et al. (Ref. 34) (points) shortening velocity at the beginning of the shortening in isotonic contractions as a function of the afterload. The fiber was prestretched from 2.0 to $2.1 \mu \mathrm{m}$. The measurements were performed at $25^{\circ} \mathrm{C}$ (Ref. 34). 


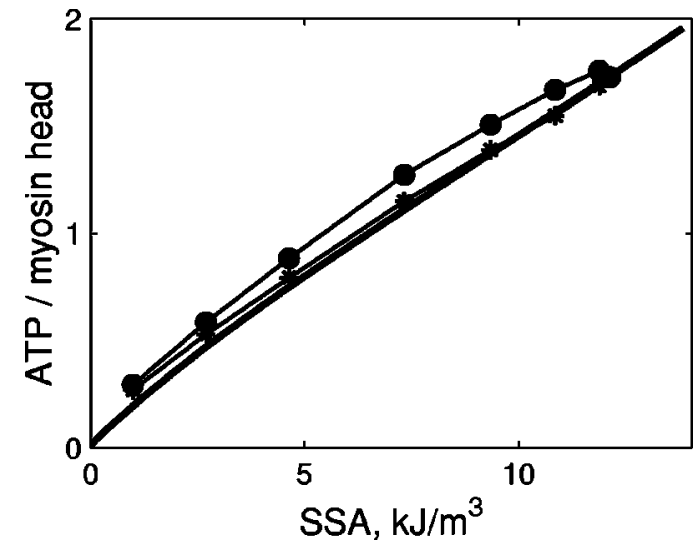

FIGURE 8. Total amount of consumed ATP molecules per myosin head during a cardiac cycle as a function of SSA for isometric (bold line), isotonic (thin solid line with circles) and the physiologic (thin solid line with stars) contractions.

cally at $2.2 \mu \mathrm{m}$ until a prescribed time $T_{r}$. At $T_{r}$, the muscle was released by reducing the externally applied force proportionally to $\exp \left\{-\left[\left(t-T_{r}\right) / 5\right]^{2}\right\}$, all units in ms. According to our simulations (Fig. 9), ATP consumption during a cycle is reduced by $38 \%$ compared to ATP consumption in the isometric contraction if the fiber is released at the time when the developed force is maximal.

It is possible that some of the cross bridges do not consume ATP for every attachment. Since the attachment reaction is reversible, there are some cross bridges that detach through reverse reaction 5 (Fig. 1) when it becomes energetically feasible. Such detachment is dependent on the contraction type and is increasing with the increase of the shortening velocity. For example, with the decrease of the afterload in the isotonic contractions, the shortening velocity increases and the relative amount of the cross bridges that detach without consuming ATP increases (Fig. 10).

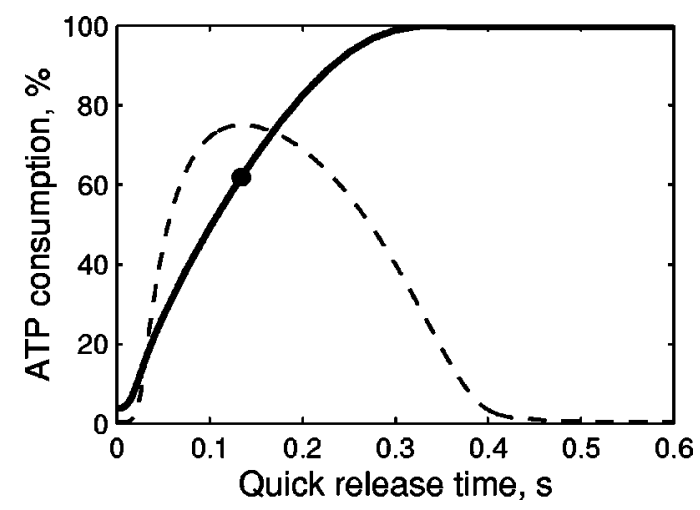

FIGURE 9. Relative amount of consumed ATP (bold line) as a function of the quick release time. The dot corresponds to the time at which the developed force during an isometric twitch (sketched by dashed line) is maximal.

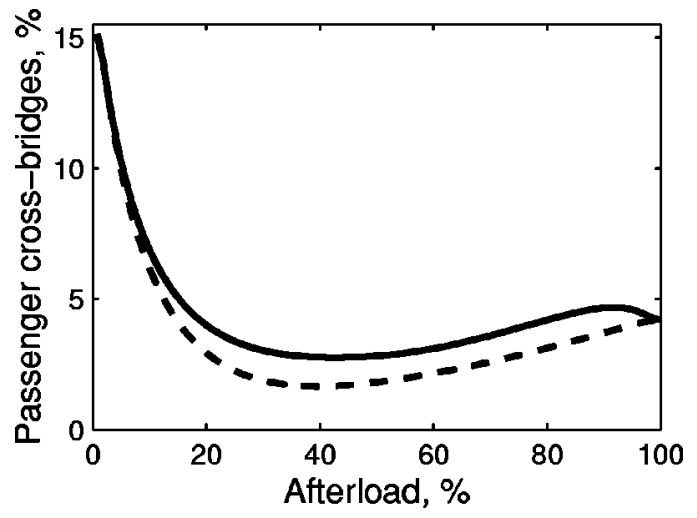

FIGURE 10. Relative amount of the passenger cross-bridges that detach without consuming ATP in isotonic (solid line) and the physiologic (dashed line) contractions as a function of the afterload during isotonic contractions.

\section{Sensitivity Analysis}

During an optimization process two functions $\left(R_{\text {act }}\right.$ and $R_{\text {cyc }}$ ) were minimized by different sets of the parameters.

The sensitivity of $R_{\text {act }}$ value to the change of the model parameters is shown in Table 2. At this analysis, the parameter and $R_{\text {act }}$ values found by optimization were used as a reference values.

The relative contribution of $R_{\text {cyc }}$ terms [see Eq. (25)] to total value of $R_{\text {cyc }}$ with the parameter values found by optimization was as follows: $R_{\text {act }} / N R_{\text {act }}$ was $4.90 \%$ of total $R_{\text {cyc }}, \quad\left(V_{0}-V_{0}^{\exp }\right)^{2} / N R_{V 0} \quad$ was $6.10 \% ， \quad\left(V_{\mathrm{ATP}}^{\text {beat }}\right.$ $\left.-V_{\text {ATP }}^{\text {SSA }}\right)^{2} / N R_{\text {ATP }}^{\text {beat }}$ was $2.12 \%, R_{l s} / N R_{l s}$ was $6.34 \%$, $R_{\mathrm{ATP}}^{t} / N R_{\mathrm{ATP}}^{t}$ was $25.73 \%, R_{\mathrm{ATP}}^{p} / N R_{\mathrm{ATP}}^{p}$ was $3.52 \%$, and $R_{\mathrm{ATP}}^{\operatorname{lin}} / N R_{\mathrm{ATP}}^{\operatorname{lin}}$ was $51.28 \%$. The sensitivity of $R_{\text {cyc }}$ to the optimization parameters (cross-bridge cycling rates) and series elasticity is summarized in Table 3.

\section{DISCUSSION}

Our mathematical model is of a self-consistent Huxley type and is in good agreement with the classical

TABLE 2. Sensitivity of the residual function $\boldsymbol{R}_{\text {act }}$ to change of model parameters.

\begin{tabular}{llc}
\hline \multicolumn{2}{c}{$\begin{array}{c}\text { Parameter } \\
\text { and its change }\end{array}$} & $\begin{array}{c}R_{\text {act }} \text { change, } \\
\%\end{array}$ \\
\hline$c_{1}$ & $+1 \%$ & +0.48 \\
$c_{1}$ & $-1 \%$ & +0.33 \\
$c_{2 M X}$ & $+1 \%$ & +0.09 \\
$c_{2 M X}$ & $-1 \%$ & +0.17 \\
$c_{2 F}$ & $+1 \%$ & +0.13 \\
$c_{2 F}$ & $-1 \%$ & +0.13 \\
$Q$ & $+1 \%$ & +0.05 \\
$Q$ & $-1 \%$ & +0.06 \\
\hline
\end{tabular}


TABLE 3. Sensitivity of the residual function $\boldsymbol{R}_{\mathrm{cyc}}$ to change of model parameters.

\begin{tabular}{|c|c|c|c|c|c|c|c|c|}
\hline \multirow[t]{2}{*}{$\begin{array}{l}\text { Parameter } \\
\text { and its change }\end{array}$} & \multicolumn{7}{|c|}{$\begin{array}{l}\text { Residual function } R_{\text {cyc }} \\
\text { term change, } \%\end{array}$} & \multirow[t]{2}{*}{$\begin{array}{c}R_{\text {cyc }} \\
\text { change, \% }\end{array}$} \\
\hline & $\frac{R_{\mathrm{act}}}{N R_{\mathrm{act}}}$ & $\frac{\left(V_{0}-V_{0}^{\text {exp }}\right)^{2}}{N R_{\mathrm{V} 0}}$ & $\frac{\left(V_{\mathrm{ATP}}^{\text {beat }}-V_{\mathrm{ATP}}^{\text {SSA }}\right)^{2}}{N R_{\mathrm{ATP}}^{\text {beat }}}$ & $\frac{R_{l s}}{N R_{l s}}$ & $\frac{R_{\mathrm{ATP}}^{t}}{N R_{\mathrm{ATP}}^{t}}$ & $\frac{R_{\mathrm{ATP}}^{p}}{N R_{\mathrm{ATP}}^{p}}$ & $\frac{R_{\mathrm{ATP}}^{\operatorname{lin}}}{N R_{\mathrm{ATP}}^{\operatorname{lin}}}$ & \\
\hline$f_{1}(+30 \mathrm{~nm})+1 \%$ & +2.88 & -0.38 & -7.52 & +1.10 & -0.24 & -0.36 & +0.27 & +0.02 \\
\hline$f_{1}(+30 \mathrm{~nm})-1 \%$ & +0.07 & +0.39 & +7.86 & -1.08 & +0.26 & +0.39 & -0.35 & +0.11 \\
\hline$f_{1}(+40 \mathrm{~nm})+1 \%$ & +3.98 & +1.16 & +2.83 & -0.25 & +0.57 & +0.86 & +0.00 & +0.57 \\
\hline$f_{1}(+40 \mathrm{~nm})-1 \%$ & -0.12 & -0.38 & -2.75 & +0.26 & -0.57 & -0.92 & -0.08 & -0.35 \\
\hline$f_{1}(+80 \mathrm{~nm})+1 \%$ & -0.66 & +6.26 & +0.68 & -3.29 & +0.58 & +1.18 & +0.41 & +0.72 \\
\hline$f_{1}(+80 n m)-1 \%$ & +1.01 & -6.07 & -0.69 & +3.31 & -0.58 & -1.06 & -0.48 & -0.45 \\
\hline$g_{1}(+\infty)+1 \%$ & -1.48 & +1.16 & +1.63 & +0.14 & -0.00 & -0.16 & +0.00 & +0.06 \\
\hline$g_{1}(+\infty)-1 \%$ & +0.37 & -0.38 & -1.64 & -0.13 & +0.00 & +0.10 & -0.07 & -0.08 \\
\hline$g_{2}(-60 \mathrm{~nm})+1 \%$ & +0.28 & +0.77 & +0.00 & +0.00 & -0.05 & -0.20 & +0.00 & +0.05 \\
\hline$g_{2}(-60 \mathrm{~nm})-1 \%$ & +0.25 & -0.77 & -0.00 & -0.00 & +0.05 & +0.20 & +0.00 & -0.02 \\
\hline$g_{2}(-0 \mathrm{~nm})+1 \%$ & +0.04 & +3.11 & +0.00 & +0.01 & -0.30 & -1.08 & +0.00 & +0.11 \\
\hline$g_{2}(-0 \mathrm{~nm})-1 \%$ & +0.63 & -3.06 & -0.00 & -0.00 & +0.31 & +1.10 & +0.00 & -0.07 \\
\hline$g_{2}(+0 \mathrm{~nm})+1 \%$ & -0.98 & +0.77 & +0.04 & -0.13 & -0.13 & -0.29 & -0.05 & -0.07 \\
\hline$g_{2}(+0 n m)-1 \%$ & -1.20 & -0.77 & -0.03 & +0.12 & +0.13 & +0.29 & -0.00 & -0.06 \\
\hline$g_{2}(+60 \mathrm{~nm})+1 \%$ & -0.37 & +1.16 & -14.93 & +0.30 & -0.91 & -2.28 & +0.22 & -0.57 \\
\hline$g_{2}(+60 \mathrm{~nm})-1 \%$ & +0.35 & -0.77 & +16.44 & -0.30 & +0.93 & +2.35 & -0.30 & +0.61 \\
\hline$f_{3}(-60 \mathrm{~nm})+1 \%$ & +4.14 & +1.55 & +0.00 & -0.02 & -0.24 & -0.81 & +0.00 & +0.22 \\
\hline$f_{3}(-60 n m)-1 \%$ & -0.28 & -1.54 & -0.00 & +0.02 & +0.24 & +0.83 & +0.00 & -0.02 \\
\hline$f_{3}(-0 \mathrm{~nm})+1 \%$ & +0.35 & +0.77 & +0.00 & -0.04 & -0.18 & -0.53 & +0.00 & +0.00 \\
\hline$f_{3}(-0 \mathrm{~nm})-1 \%$ & -0.61 & -0.77 & -0.00 & +0.04 & +0.18 & +0.53 & +0.00 & -0.01 \\
\hline$f_{3}(+0 \mathrm{~nm})+1 \%$ & +0.55 & +0.00 & +1.69 & +0.81 & -0.89 & +0.16 & +0.00 & -0.16 \\
\hline$f_{3}(+0 \mathrm{~nm})-1 \%$ & -1.61 & +0.00 & -1.62 & -0.77 & +0.90 & -0.15 & -0.05 & +0.09 \\
\hline$f_{3}(+60 \mathrm{~nm})+1 \%$ & +0.81 & +0.77 & -55.10 & -3.00 & -3.59 & -7.92 & +0.68 & -2.63 \\
\hline$f_{3}(+60 n m)-1 \%$ & -1.77 & -0.00 & +77.80 & +3.13 & +3.72 & +9.58 & -0.75 & +3.28 \\
\hline$I_{\mathrm{se}}+1 \%$ & +0.41 & -20.42 & -4.90 & +14.21 & -12.65 & -19.95 & +33.01 & +7.87 \\
\hline$I_{s e}+10 \%$ & +1.37 & -20.76 & -13.89 & +14.09 & -12.35 & -21.87 & +35.23 & +8.59 \\
\hline$I_{\text {se }}+100 \%$ & -4.52 & +26.33 & -64.43 & +13.42 & -9.92 & -34.42 & +55.21 & +19.47 \\
\hline$I_{s e}-1 \%$ & -0.36 & -20.42 & -2.77 & +14.22 & -12.70 & -19.50 & +32.53 & +7.68 \\
\hline$I_{s e}-10 \%$ & -0.83 & -19.73 & +7.43 & +14.35 & -13.00 & -17.42 & +30.35 & +7.06 \\
\hline$I_{s e}-50 \%$ & -1.22 & -18.69 & +66.07 & +14.90 & -14.72 & -7.00 & +22.10 & +5.07 \\
\hline
\end{tabular}

measurements of SSA and oxygen consumption dependency. As has been measured in different laboratories, oxygen (or ATP) consumption depends linearly on SSA (or its full-organ analog PVA). It has been shown in several experimental studies that this dependency is the same for the isometric and shortening contractions. The model presented here reproduces this property of the cardiac muscle very well (see Fig. 8).

In this work we found the set of model parameters that gave the best fit between model solution and the experiment. As the result of the optimization, the model solution is in good agreement with the behavior of the cardiac muscle in isometric and shortening contractions. First, the contractile machinery efficiency computed by the model is in good agreement with the experimental data. Taking the efficiency of the oxidative phosphorylation to the free energy change of ATP hydrolysis equal to $70 \%$ and the contractile efficiency from the excess of $\mathrm{VO}_{2}$ to PVA of $35 \%-47 \%$, the cross-bridge contractile efficiency computed from the experimental data is $50 \%-67 \% .^{10,27}$ In our simulations, the cross-bridge con- tractile efficiency is $65 \%$. Second, the computed isometric active stress during a beat replicates well the measured stress in the isosarcometric contraction at different sarcomere length values (Fig. 3). Third, the contraction duration is smaller in the isotonic case if compared with the isometric case (Fig. 4) which reproduces the typical isotonic contraction experiment results. ${ }^{2}$ Fourth, the endsystolic point in the stress-strain diagram in the isotonic contraction lies on the end-systolic line computed for the isometric case. According to the corresponding measurements performed by Hisano and Cooper IV, ${ }^{14}$ endsystolic lines are different for isometric and isotonic contractions and depend on the loading conditions. However, these lines are relatively close to each other.

We tried to predict some of the muscle properties of the model without any fitting. Namely, we have not fitted the shortening velocity dependency on the afterload (Fig. 7) except the velocity at afterload of $2.5 \mathrm{kPa}$, ATP consumption in a cycle in quick release experiments (Fig. 9), and isotonic contraction duration (Fig. 4). All these 
model predictions are in good agreement with the corresponding measurements. The largest difference detected between model prediction and the measurements, is the difference in sarcomere shortening velocity between the experimental study ${ }^{34}$ and the model solution at low afterloads. However, at afterloads larger than $10 \mathrm{kPa}$ the computed and measured shortening velocities are in good agreement with each other.

ATP consumption in the quick release experiment computed by the model correlates well with the measurements by Hisano and Cooper IV. ${ }^{14}$ Namely, these authors have found that the oxygen consumption in a cycle drops by $36 \pm 4 \%$ when the muscle is released at the time of peak force. Taking into account that the basal level consumption was about $12 \%$ in isometric contraction, ${ }^{14}$ the drop in ATP consumption by the crossbridges is about $41 \pm 5 \%$ if the basal consumption is the same for isometric and quick-released contractions. According to our simulations, the drop is about $38 \%$ which is close to the measurements.

According to the model sensitivity analysis, summarized in Table 3, the residual function $R_{\text {cyc }}$ minimized during optimization can be reduced further if the optimization stopping criteria would be ignored. Namely, the changes in cross-bridge cycling rates can lead to $R_{\text {cyc }}$ reduction. The largest drop of $R_{\text {cyc }}$ is expected if $f_{3}$ $(+60 \mathrm{~nm})$ is increased. One can use the information obtained from the sensitivity analysis to reduce the largest difference between the model predictions and measurements - the sarcomere shortening velocity at low afterloads. To reduce this difference, one can reduce $f_{1}(+80 \mathrm{~nm})$ or $g_{2}(-0 \mathrm{~nm})$. The reduction of $f_{1}(+80$ $\mathrm{nm})$ seems to be the best choice since it reduces $R_{\text {cyc }}$ much more than reduction of $g_{2}(-0 \mathrm{~nm})$. Table 3 shows the influence of the compliance within thick and thin filaments $\left(l_{\mathrm{se}}\right)$ to some of the model properties. According to our results, the change of $l_{\mathrm{se}}$ has the largest impact to sarcomere shortening velocity at low afterload and dependence of ATP consumption on SSA. However, taking into account that even the doubling and reduction by two of $l_{\text {se }}$ does not change the order of $R_{\text {cyc }}$, we suggest that it is possible to find the set of the model parameters with different $l_{\text {se }}$ values which will fit the experimental data as well. The compliance of the filaments described by $l_{\text {se }}$ is the compliance of only the nonoverlap zone. Due to the limitations of the Huxley-type model, we had to ignore the compliance within the overlap zone of the filaments. $^{5}$

The correlation between the different experimental measurements and the computations adds support to the model predictions. One important model prediction is that the ATP consumption rate within a beat depends strongly on the contraction type. For example, in the isotonic contractions a large amount of ATP is consumed by the cross-bridges in the beginning of the cycle. On the other hand, ATP consumption in isometric contraction is spread more homogeneously along a beat (see Fig. 5). Thus, the differences in the measurements of the oxygen consumption in the quick-release experiments outlined by Taylor et al. ${ }^{30}$ may be explained by the differences in the muscle shortening dynamics at the sarcomere level between isovolumetric heart contractions ${ }^{8}$ and isometric isolated papillary muscle contractions. ${ }^{14}$ This prediction is in concord with the high sensitivity of the ATP consumption rate profile on the parameters of the Huxley-type model shown by Taylor et al. ${ }^{30}$

One of the results obtained by our model, is the relatively small amount of the passenger cross bridges in isotonic contractions. In a theoretical study of the skeletal muscle by Cooke et al., ${ }^{4}$ the number of cross bridges that detached without hydrolyzing ATP was up to $80 \%$ at high contraction velocities. In our simulations the number of passenger cross bridges were not more than $15.2 \%$ during a cycle (Fig. 10), or more than 5 times smaller. This difference may indicate the differences in the muscle type or in the contraction protocol. However, the relatively small difference between amount of the passenger cross bridges in isotonic and in the physiologic contractions indicate that only the muscle shortening phase of the contraction is important since the muscle lengthening phase of the contraction is different for these contractions.

This study is not the first one which uses the Huxleytype models to simulate mechanochemical properties of the muscle. A series of studies by Pate and Cooke on the skeletal muscle fiber mechanics and biochemistry has shown a theoretical way to explain high economy of the rapidly contracting muscle. ${ }^{4}$ We have used a similar approach and added a $\mathrm{Ca}^{2+}$-activation description to our model which is required in the simulations of cardiac muscle contraction during a beat. The composition of the $\mathrm{Ca}^{2+}$-activation model together with application of the Hill-Eisenberg formalism helped us to overcome the difficulties experienced in several theoretical studies ${ }^{29,31}$ of cardiac oxygen consumption such as nonlinear oxygen consumption dependency on SSA and large differences in this dependency for the isometric and isotonic contractions.

Most of the cardiac muscle activation properties included in our model have been previously used by others: the reduction of the deactivation rate constant $c_{2}$ with the increase sarcomere length [see Eqs. (18) and (19)] at the constant activation rate $c_{1} ;{ }^{23}$ the cooperativity of the $\mathrm{Ca}^{2+}$ binding to the troponin $\mathrm{C}$ [see Eq. (18) $].^{20}$ However, we assumed that duration of troponin $\mathrm{C}$ activation depends on the sarcomere length [see Eqs. (20) and (21)]. It is important to note that intracellular $\mathrm{Ca}^{2+}$ concentration transient duration is not influenced by sarcomere length significantly, ${ }^{19}$ but taking into account the complex multistep interaction between $\mathrm{Ca}^{2+}$ 
and troponin $\mathrm{C}^{7}$ we proposed that the activation parameter $A$ stands for the activated troponin $C$ and $B$ for the intermediate state in reaction between troponin $\mathrm{C}$ and $\mathrm{Ca}^{2+}$. Thus, we assumed that the intermediate state between inactivated and activated troponin $\mathrm{C}$ depends on the sarcomere length. Since our model is of a phenomenological type and in this paper we are not interested in the precise description of $\mathrm{Ca}^{2+}$ dynamics, we predescribed the intermediate state dependency on time and sarcomere length to compute stress, deformation, and ATP consumption of the muscle.

Recently, Landesberg and Sideman ${ }^{21}$ reproduced with their cross-bridge model the linear relationship between ATP consumption and SSA in isometric contraction. They have shown that their model is able to reproduce the linear relationship only if the cooperativity between the cross-bridge recruitment and the generated force is assumed. By making this assumption, Landesberg and Sideman $^{21}$ prolonged the twitch duration at the larger sarcomere length values. Taking into account the SSA dependency on sarcomere length, such prolongation is required to reproduce the relationship between ATP consumption and SSA. Namely, one can roughly estimate that the maximal developed force is increasing linearly as a function of sarcomere length, and consequently, SSA is increasing with the second order of the sarcomere length. To have the same second-order increase of ATP consumption, which is the time integral of ATP consumption rate in a beat, the twitch duration has to increase linearly with respect to sarcomere length since maximal ATP consumption rate is increasing almost proportionally to maximal developed stress. In our model, we prolonged the twitch duration by prolonging troponin $\mathrm{C}$ activation with the increase of sarcomere length. The cooperativity of the $\mathrm{Ca}^{2+}$ binding to troponin $\mathrm{C}^{32}$ used in this paper differs from the mechanism used by Landesberg and Sideman. ${ }^{21}$ The cooperativity mechanism was introduced into the model to reduce the generated force faster at the end of the twitch. Without the cooperativity mechanism, the computed twitch duration was much longer than measured. Thus, there are considerable differences between descriptions of the muscle activation mechanism used by us and by Landesberg and Sideman. ${ }^{21}$ However, taking into account that, opposite to Landesberg and Sideman, ${ }^{21}$ no additional insight in the cardiac muscle activation dynamics is sought in this work, and the simplified approach used is justified.

We modeled the active force dependency on sarcomere length only through the muscle activation and neglected the influence of the real structure of the sarcomere, such as double-overlap regions, to the force-length dependency. This is common practice in the cardiac muscle modeling ${ }^{23,29,31}$ and it is based on a high sensitivity of $\mathrm{Ca}^{2+}$-induced activation to the value of the sarcomere length. ${ }^{18}$ This model simplification is impor- tant if one assumes, like Landesberg and Sideman ${ }^{21}$ did, that the cross bridges consume ATP but do not produce any mechanical force in the double-overlap region of the thick and thin filaments. If true, our efficiency computations are not correct since we neglected ATP consumption by cross bridges in the double-overlap regions. The relative amount of cross bridges in the doubleoverlapped region (and according to the assumption of Landesberg and Sideman ${ }^{21}$ an error that we introduce) is a linear function of the sarcomere length which declines with the increase of the sarcomere length. Assuming that thick filament length is $1.5 \mu \mathrm{m}$, the thin filament length is $1.05 \mu \mathrm{m}, Z$-line width is $0.1 \mu \mathrm{m}$, width of "bare" area in the center of thick filament is $0.1-0.2 \mu \mathrm{m}$, the relative amount of cross bridges in the doubleoverlapped region is less than $1 / 5$ at sarcomere length larger than 1.8 or $1.7 \mu \mathrm{m}$ for the bare area width of 0.1 or $0.2 \mu \mathrm{m}$, respectively. Thus, the introduced error is reasonably small at sarcomere length values larger than $1.8 \mu \mathrm{m}$. Finally, we would like to stress that this error estimation of computed cross-bridge contractile efficiency is based on the assumption that cross bridges consume ATP and do not produce any force in the double-overlap region. Regardless to the intriguing experimental studies outlined by Landesberg and Sideman ${ }^{21}$ in proof of their assumption, to the best of our knowledge there is no experimental study that directly shows such a behavior of the cross bridges.

From the comparison of our study with the theoretical studies performed before, we conclude that the main difference which allowed us to replicate the linear dependency of ATP consumption by cross bridges on SSA was the approach we used to obtain the model parameters. Namely, one of the requirements used in the model parameters estimation was the linear dependency between ATP consumption and SSA in isometric and shortening contractions. Thus, we treated the relationship between ATP consumption and SSA as a fundamental property of the muscle and found the cross-bridge cycling rate constants and the activation parameters using this macroscopical property of the muscle. It is important to note, that the set of the parameters found by optimization may not be unique. For example, one can find the cross-bridge rates with different dependencies on $z$ and still obtain good results. In this work, we tried to find the rates with as simple shapes as possible to fit the desired data.

To summarize, we have replicated the experimentally observed linear relation between cardiac ATP consumption and stress-strain area with a model, composed of a three-state Huxley-type model for cross-bridge interaction and a phenomenological model of $\mathrm{Ca}^{2+}$-induced activation. The simulation results demonstrated that this dependency does not require a high number of cross bridges which detach without consuming ATP. 


\section{ACKNOWLEDGMENTS}

The authors are grateful to Dr. G. J. Stienen, the Free University of Amsterdam, the Netherlands, and Dr. O. Kongas, National Institute of Chemical Physics and Biophysics, Estonia, for stimulating discussions. This work was partly supported by the Netherlands Organisation for International Cooperation in Higher Education, and Estonian Science Foundation (Grant No. 3739).

\section{REFERENCES}

${ }^{1}$ Bovendeerd, P. H., T. Arts, J. M. Huyghe, D. H. van Campen, and R. S. Reneman. Dependence of local left ventricular wall mechanics on myocardial fiber orientation: a model study. J. Biomech. 25:1129-1140, 1992.

${ }^{2}$ Brutsaert, D. L., N. M. de Clerck, M. A. Goethals, and P. R. Housmans. Relaxation of ventricular cardiac muscle. $J$. Physiol. (London) 283:469-480, 1978.

${ }^{3}$ Callewaert, G. Excitation-contraction coupling in mammalian cardiac cells. Cardiovasc. Res. 26:923-932, 1992.

${ }^{4}$ Cooke, R., H. White, and E. Pate. A model of the release of myosin heads from actin in rapidly contracting muscle fibers. Biophys. J. 66:778-788, 1994.

${ }^{5}$ Daniel, T. L., A. C. Trimble, and P. B. Chase. Compliant realignment of binding sites in muscle: transient behavior and mechanical tuning. Biophys. J. 74:1611-1621, 1998.

${ }^{6}$ Delhaas, T., T. Arts, F. W. Prinzen, and R. S. Reneman. Regional fibre stress-fibre strain area as an estimate of regional blood flow and oxygen demand in the canine heart. $J$. Physiol. (London) 477:481-496, 1994.

${ }^{7}$ Dong, W., S. S. Rosenfeld, C. K. Wang, A. M. Gordon, and H. C. Cheung. Kinetic studies of calcium binding to the regulatory site of troponin $\mathrm{C}$ from cardiac muscle. J. Biol. Chem. 271:688-694, 1996.

${ }^{8}$ Duwel, C. M., and N. Westerhof. Feline left ventricular oxygen consumption is not affected by volume expansion, ejection or redevelopment of pressure during relaxation. Pflugers Arch. 412:409-416, 1988.

${ }^{9}$ Eisenberg, E., T. L. Hill, and Y. Chen. Cross-bridge model of muscle contraction. Quantitative analysis. Biophys. J. 29:195-227, 1980.

${ }^{10}$ Gibbs, C. L., and C. J. Barclay. Cardiac efficiency. Cardiovasc. Res. 30:627-634, 1995.

${ }^{11}$ Gibbs, C. L., and J. B. Chapman. Cardiac mechanics and energetics: chemomechanical transduction in cardiac muscle. Am. J. Physiol. 249:H199-H206, 1985.

${ }^{12}$ Goldman, Y. E. Kinetics of the actomyosin ATPase in muscle fibers. Annu. Rev. Physiol. 49:637-654, 1987.

${ }^{13}$ Hill, T. L. Theoretical formalism for the sliding filament model of contraction of striated muscle. Part I. Prog. Biophys. Mol. Biol. 28:267-340, 1974.

${ }^{14}$ Hisano, R., and G. Cooper. IV. Correlation of force-length area with oxygen consumption in ferret papillary muscle. Circ. Res. 61:318-328, 1987.

${ }^{15}$ Huxley, H. E. Sliding filaments and molecular motile systems. J. Biol. Chem. 265:8347-8350, 1990

${ }^{16}$ Irving, M. Weak and strong crossbridges. Nature (London) 316:292-293, 1985.

${ }^{17}$ Janssen, P. M. and W. C. Hunter. Force, not sarcomere length, correlates with prolongation of isosarcometric contraction. Am. J. Physiol. 269:676-685, 1995.

${ }^{18}$ Jewell, B. R. A reexamination of the influence of muscle length on myocardial performance. Circ. Res. 40:221-230, 1977.

${ }^{19}$ Lakatta, E. G., Length modulation of muscle performance: Frank-Starling law of the heart. In: H. A. Fozzard, E. Haber, R. B. Jennings, A. M. Katz, and H. E. Morgan, eds., The Heart and Cardiovascular System, New York: Raven, 2nd ed., 1992, pp. 1325-1351.

${ }^{20}$ Landesberg, A., and S. Sideman. Coupling calcium binding to troponin $\mathrm{C}$ and cross-bridge cycling in skinned cardiac cells Am. J. Physiol. 266:1260-1271, 1994.

${ }^{21}$ Landesberg, A., and S. Sideman. Regulation of energy consumption in cardiac muscle: analysis of isometric contractions. Am. J. Physiol. 276:H998-H1011, 1999.

${ }^{22}$ Moré, J. J., D. C. Sorensen, K. E. Hillstrom, and B. S. Garbow. The MINPACK project. In: W. J. Cowell, ed., Sources and Development of Mathematical Software. Englewood Cliffs, NJ: Prentice-Hall, 1984.

${ }^{23}$ Panerai, R. B. A model of cardiac muscle mechanics and energetics. J. Biomech. 13:929-940, 1980.

${ }^{24}$ Pate, E., and R. Cooke. A model of crossbridge action: the effects of ATP, ADP and Pi. J. Muscle Res. Cell Motil. 10:181-196, 1989.

${ }^{25}$ Rijcken, J., P. H. Bovendeerd, A. J. Schoofs, D. H. van Campen, and T. Arts. Optimization of cardiac fiber orientation for homogeneous fiber strain at beginning of ejection. $J$. Biomech. 30:1041-1049, 1997.

${ }^{26}$ Saeki, Y. Crossbridge dynamics under various inotropic states in cardiac muscle: evaluation by perturbation analyses. Jpn. J. Phys. 45:687-705, 1995.

${ }^{27}$ Suga, H. Ventricular energetics. Physiol. Rev. 70:247-277, 1990.

${ }^{28}$ Taylor, E. W. Mechanism and energetics of actomyosin ATPase. In: H. A. Fozzard, E. Haber, R. B. Jennings, A. M. Katz, and H. E. Morgan, eds., The Heart and Cardiovascular System, New York, Raven, 2nd ed., 1992.

${ }^{29}$ Taylor, T. W., Y. Goto, K. Hata, T. Takasago, A. Saeki, T. Nishioka, and H. Suga. Comparison of the cardiac force-time integral with energetics using a cardiac muscle model. $J$. Biomech. 26:1217-1225, 1993.

${ }^{30}$ Taylor, T. W., Y. Goto, K. Hata, T. Takasago, A. Saeki, T. Nishioka, and H. Suga. Cardiac quick-release contraction mechanoenergetics analysis using a cardiac muscle crossbridge model. Am. J. Physiol. 268:H2544-H2552, 1995.

${ }^{31}$ Taylor, T. W., Y. Goto, and H. Suga. Variable cross-bridge cycling-ATP coupling accounts for cardiac mechanoenergetics. Am. J. Physiol. 264:H994-1004, 1993.

${ }^{32}$ Tobacman, L. S., and D. Sawyer. Calcium binds cooperatively to the regulatory sites of the cardiac thin filament, $J$. Biol. Chem. 265:931-939, 1990.

${ }^{33}$ van der Velden, J., A. F. Moorman, and G. J. Stienen. Agedependent changes in myosin composition correlate with enhanced economy of contraction in guinea-pig hearts. $J$. Physiol. (London) 507:497-510, 1998

${ }^{34}$ van Heuningen, R., W. H. Rijnsburger, and H. E. ter Keurs. Sarcomere length control in striated muscle. Am. J. Physiol. 242:H411-H420, 1982.

${ }^{35}$ Zahalak, G. I., and S. P. Ma. Muscle activation and contraction: constitutive relations based directly on cross-bridge kinetics. J. Biomech. Eng. 112:52-62, 1990. 